\title{
FROM PRODUCTION TO SELECTION OF INTERPRETATIONS FOR NOVEL CONCEPTUAL COMBINATIONS: A DEVELOPMENTAL APPROACH
}

\author{
SANDRA JHEAN-LAROSE ${ }^{\mathrm{a}}-$ BRUNO LECOUTRE $^{\mathrm{b}}$ \\ - GUY DENHIÈRE ${ }^{a}$ \\ ${ }^{a}$ Université d'Orléans, EA 4004 CHArt, 'Cognitions Humaine et Artificielle', France \\ ${ }^{\mathrm{b}}$ ERIS, Laboratoire de Mathématiques Raphaël Salem, France \\ Corresponding author: Sandra Jhean-Larose, address: 41 Rue Gay Lussac, \\ 75005 Paris, France, e-mail: sandra.jhean-larose@ephe.sorbonne.fr
}

\begin{abstract}
This study looks at how combinations of two French nouns are interpreted. The order of occurrence of the constituents of two types of conceptual combinations, relation and property, was manipulated in view of determining how property-based and relation-based interpretations evolve with age. Three groups of French-speaking children (ages 6, 8, and 10) and a group of adults performed an interpretation-selection task. The results for the children indicated that while property-based interpretations increased with age, relation-based interpretations were in the majority for both combination types, whereas for the adults, relation-based interpretations were in the minority for property combinations. For the children and adults alike, the most frequent interpretations were ones in which the head noun came first and was followed by the modifier (the opposite of the order observed for English).
\end{abstract}

Keywords: conceptual combination, relation, property, interpretation, development

\section{Introduction}

A large part of human cognition involves combining concepts of variable familiarity in order to build new concepts (Murphy 2002; Le Ny 2005). Within the past few years, research in English has studied this concept-combining process by looking at pairs of nouns (N1 N2) in which the first noun acts as the modifier of the second (Costello-Keane 2000; 2001; Estes-Glucksberg 1999; 2000; Gagné-Shoben 1997; Hampton 1987; Medin-Shoben 1988; Murphy 1988; 1990; 2002; Wisniewski 1997; Wisniewski-Middleton 2002). In French, new noun-noun combinations 
are being used more and more often today, especially in spoken and written journalism. French expressions like plan retraite ${ }^{1}$ ('plan retirement' for retirement account), ${ }^{2}$ soirée pizza ('evening pizza' for pizza party), école pilote ('school pilot' for pilot school), plan école ('plan school' for school program), université cocon ('university cocoon' for cocoon university), nageuse tortue ('swimmer turtle' for swimming turtle), humanitaires mercenaires ('humanitarians mercenaries' for mercenary humanitarians), amour Kleenex ('love Kleenex' for disposable love) are just a few examples of expressions currently being heard or read. In spite of their peculiar syntax, such expressions are apparently not causing any real interpretation problems, and this phenomenon had already become sufficiently widespread in French for a book about these so-called 'epithet substantives' to be published as early as 1990 (Noailly 1990). Noailly states:

\footnotetext{
"We are witnessing one of the principal signs of the beginnings of a syntactic mutation of French: having come to the end of its 'analytic' route, our language may now be coming back up that same road and trying itself out on more abrupt, more basic, and more immediate syntactic forms, with fewer articles, fewer suffixes, fewer prepositions." (op.cit., 13, our translation)
}

Due to their frequency in the language, some noun-noun combinations have become lexicalized as hyphenated compound nouns and are now found in dictionaries, e.g., chèque-repas ('check-meal' for meal ticket), homme-grenouille ('man-frog' for frogman), micro-trottoir ('mic-sidewalk' for street interview), etc.

Experimental psychology studies on the interpretation of novel nounnoun combinations have focused on either the construal of a relationship between the constituents (N1 and N2) or the transfer of a property from one constituent to the other (Jhean-Larose-Denhière 2006; 2007). For instance, the combination chocolate elephant is usually interpreted in terms of the relation 'made of' (an elephant made of chocolate) rather than as a brown-colored elephant, whereas elephant garlic usually triggers the interpretation 'a large head of garlic' rather than, say, 'garlic that looks like an elephant'/'garlic for elephants' (Wisniewski 1997; Wisniewski-

${ }^{1}$ In "proper" French grammar, plan retraite, for example, would be plan de retraite. Note that the syntax of noun phrases in French is the opposite of English, i.e., the French noun precedes its modifiers, as in livre intéressant 'book interesting'.

${ }^{2}$ For each French combination, a word-for-word English translation is shown in quotes, followed by a rough translation of the meaning when applicable. 
Love 1998). Elephant garlic is a familiar term - it is the actual name used for a particular kind of garlic.

Gagné and Shoben (1997) suggested that people relate the two nouns to each other based on their linguistic experience. These authors postulate that the way a given combination is interpreted is determined by implicit stored knowledge of the frequency of relations. The theoretical claim is that knowledge about how the modifier tends to be used plays a role. One way to define this more general concept is by frequency of relations. This principle is the basis of their CARIN model (Competition Among Relations In Nominals). For instance, the modifier 'mountain' is typically associated with the 'location' relation (Levi (1978)), as in the combinations mountain stream, mountain resort, and mountain goat. Experimental results supporting this model indicate that only the frequency of relations associated with the modifier (N1) have an impact on interpretation time. Gagné and Shoben (1997) explain that the modifier may have a greater influence than the head noun because (i) it determines the interpretation of the combined concept and because (ii) it is encountered first, and consequently, frequent relations associated with the modifier are activated before frequent relations associated with the head noun. If this is so, the influence of the modifier is simply due to the order in which the nouns occur: in English, the modifier always precedes the head noun. The authors also propose that the modifier might "be the more influential component in that it is used to denote a particular kind of instance belonging to the head-noun category" (ibid., 73). Thus, Gagné and Shoben offer both an order-based explanation and one based on the particular role played by the constituents - of course, they could not differentiate between these two possibilities because the study was conducted in English (which confounds these two situations). However, it is impossible to rule out the possibility that the modifier (N1) also has certain "semantic privileges" (Estes-Glucksberg 1999; 2000).

To separate effects due to the order of the constituents from those that can be ascribed to their semantic nature, Storms and Wisniewski (2005) studied the interpretation of noun-noun combinations in Indonesian, a language where the two constituents are in the opposite order to that of English. These authors conducted two experiments using a list of 57 combinations taken from Gagné and Shoben's material (1997, Experiment 1). In the first experiment, Storms and Wisniewski asked participants to produce one of the two constituents in a combination in which only the modifier (chocolate $X$ ?) or only the head noun ( $X$ ? crisis) 
was given, and calculated the frequency of the relations associated with each term. In their second experiment, participants had to choose a meaning for 57 combinations divided into four categories obtained by crossing the relation-frequency factor (high vs. low) and the type-of-constituent factor (head noun vs. modifier). As a whole, the results confirmed the critical role of the modifier in the interpretation of novel combinations. These findings are in line with the CARIN model, which states that only the frequency of the relation associated with the modifier has an effect on response time (Gagné-Shoben 1997), and they support the hypothesized "semantic privilege" of the modifier.

Unlike the CARIN model, Wisniewski's (1996; 1997) dual-process model stipulates that highly similar constituents often lead to interpretations resulting from the transfer of a property of the modifier (N1) to the head noun (N2). In this model, relation-based interpretations are thought to be used when there exists a plausible scenario that can link the two concepts to each other (Caramelli-Borghi 2004), as in city kid, where a plausible scenario could be 'a child that lives in the city'. When the constituents are similar, it is difficult to link them via a plausible scenario because their roles tend to be alike. For instance, the combination whiskey beer cannot be interpreted on the basis of the relation 'is a drink' because both constituents are plausible candidate fillers for the same role (Wisniewski-Love 1998, 180). A plausible way of interpreting whiskey beer consists of selecting a property of whiskey and transferring it to beer to produce an interpretation something like: 'a beer that tastes like whiskey'. Wisniewski and Middleton (2002) suggested that property-transfer interpretations go through a three-step process. First, the semantic structures of the two constituents are compared ("aligned"). Next, a salient property that distinguishes the two structures is selected. Then the selected property is incorporated into the head noun (see Jhean-Larose-Denhière $2006,283) .{ }^{3}$ For the combination elephant garlic, the property 'big' of N1 is modified and adjusted to fit with the characteristics of N2, 'garlic'.

In sum, while Gagné and Shoben (1997) contend that the frequency of relations associated with the modifying noun (N1), stored in people's memory, is the main factor that evokes a relation and determines the interpretation made of a conceptual combination, Wisniewski $(1996 ; 1997)$

3 The comparison and alignment process was initially evoked in models of metaphors and analogies (Bowdle-Gentner 2005; Gentner 1983; 1989; Glucksberg et al. 1997; Markman-Gentner 1993; Markman-Wisniewski 1997; WolffGentner 2000; for a review, see Gineste-Scart-Lhomme 1999). 
postulates that semantic similarity between constituents determines the interpretation of a combination via a semantic relation in cases of low similarity, and via property transfer in cases of high similarity. The constituent-similarity effect is a key issue in the study by Parault et al. (2005). Their main goal was to find out whether 6- and 9-year-old children (as compared to adults) use conceptual similarity as a basis for interpreting new conceptual combinations, and whether these children are capable of making interpretations in terms of properties and relations. The authors used 36 conceptual combinations, 16 similar combinations borrowed from Wisniewski (1996), and 16 dissimilar combinations and 4 non-interpretable combinations borrowed from Gagné and Shoben (1997). The interpretations produced were classified into two categories: relationbased (e.g., 'a germ that's in milk' and 'a headache you get in college' in response to milk germ and college headache, respectively) and propertybased (e.g., 'a hotel where you can live' and 'a bicycle with a motor' in response to apartment hotel and motorcycle bicycle). Conceptual similarity was found to have an impact among both children and adults. Like the adults, the children interpreted the highly similar combinations in terms of a property and the dissimilar combinations in terms of a relation. However, compared to the adults, the 6- and 9-year-olds had more trouble interpreting the similar combinations than the dissimilar ones; the children were aware that relation-based interpretations were not suitable for highly similar combinations, but they were incapable of coming up with another type of interpretation. Referring to Wisniewski's (1996, 1997) model, Parault et al. (2005) suggested that children are capable of aligning the properties of the two constituents (step 1) but they have difficulty both in selecting a property of the modifier to transfer (step 2) and in incorporating that property into the head noun (step 3). The authors concluded that at these ages, children are in the process of learning to select a property of the first constituent and to integrate it into the second constituent.

In order to obtain further data on the ability of French children and adults to make relation- or property-based interpretations of conceptual combinations likely to trigger one rather than the other of these two kinds of interpretation, Jhean-Larose et al. (2009) conducted a study using an interpretation-production task involving 24 novel combinations (12 relation combinations and 12 property combinations) borrowed from Gagné and Shoben's (1997) corpora and translated into French. The study was also aimed at examining the respective roles played by each constituent 
of a conceptual combination. In English, the respective locations of the constituents, i.e., modifier N1 followed by head noun N2 (fish sandwich), does not allow one to unequivocally ascribe the most frequent interpretation - namely, modifier (Mod) followed by head noun (Head) - to the "semantic nature" of the constituent or to its location in the noun-noun combination (Gagné-Shoben 1997; Storms-Wisniewski 2005). Unlike English, the head noun in French is usually located before the modifier, as in sandwich poisson ('sandwich fish' for fish sandwich). We manipulated the location of the modifier and head noun in combinations taken from Gagné and Shoben's (1997) lists in order to determine how a constituent's location (first or second position) and semantic nature ('modifier' or 'head noun') affect the interpretation of relation combinations such as brûlure incendie ('burn fire') and property combinations such as boisson épingle ('drink pin'). Native French-speaking 6-, 8-, and 10-year-old children and adults were tested.

The results obtained for the type of interpretation indicated an effect of the location of constituent N2. N2N1 interpretations significantly outnumbered N1N2 interpretations, with a greater difference between the two for combinations presented in the order that corresponded to the dominant interpretation N2N1. This finding was obtained massively for adults and children alike, and for both relation and property combinations. It is compatible with Noailly (1990) view according to which French syntax places the modified noun before its modifiers, and lexical memory dictates the usage of each particular nominal lexeme. For this author, "Nouns are imbalanced in terms of their nominal or adjectival usage. The decoding process is assumed to involve assessing, estimating, and weighing the relative tendencies of each one of the two adjacent nouns" (op.cit., 28, our translation). It is up to the psychologist to determine what cognitive factors, during development, are responsible for the changes observed. Answering this question in terms of Gagné and Shoben's (1997) CARIN model consists of postulating that the relational frequency of one of the two constituents - the modifier in English, the head noun in French according to Jhean-Larose et al.'s (2009) results - determines the most probable interpretation of the combination. Note that in both English and French, it is the noun located in first position that determines the interpretation and acts as the governing noun (Jhean-Larose et al. 2007).

Regarding whether conceptual-combination interpretations are based on finding a relation between the two constituents or on transferring a 
property from one to the other, the results indicated that regardless of constituent order, relation-based interpretations were always in the majority; they were less numerous for adults than for children; and they decreased in frequency as the children grew older. However, this overall tendency masked an important difference between children and adults: the children gave more relation-based responses for both relation and property combinations, whereas the adults, while doing likewise for relation combinations, more often made property-transfer interpretations for property combinations. In addition, among the children, the difference between the proportions of relation and property-based responses increased with age.

These results are compatible with the CARIN theory (Gagné-Shoben 1997). According to this theory, all combined concepts are based on a relational structure. This relational structure could give rise to property interpretations under some cases (Gagné 2000). Unlike the dual-process theory (Wisniewski 1996; 1997), Gagné and Shoben do not argue that property-interpretations are based on a fundamentally separate and distinct process. Instead, the CARIN theory relation-linking and property-mapping could both occur (e.g., the relation facilitates the mapping of properties that are relevant to that particular relation). This explanation is consistent with the response time data reported in Gagné (2000) in which it took longer to respond to property interpretations than to relation interpretations. In contrast, the dual-process theory argues that either relation-linking or property-mapping occurs and, consequently, that these two processes are independent. If the specification of properties occurs after the selection of a relation (as proposed in Gagné 2000), then older children might have greater knowledge about the concepts involved and hence be more likely to identify particular properties in their response. This would occur due to elaboration of the concepts following the construction of an initial relation-based combined concept (see also Murphy 1990 for an alternative take of the process of elaboration).

In view of determining why children produce fewer property interpretations than adults and how much of this is due to inaccessibility of the concepts, we devised an experimental device that facilitates this task. Rather than being asked to come up with an interpretation, the children had to choose which of four interpretations "suited them the best", the one they "preferred". In other words, our aim was to determine whether the age-related differences in production observed among children are primarily rooted in differences in the availability or accessi- 
bility of the concepts in memory (see Tiberghien 1997). Indeed, in tasks involving producing interpretations of combinations, the effects of two factors are inextricably confounded in the final product: (i) availability in memory of the concepts and their properties, resulting from the fact that children have built more or less elaborate mental representations of the concepts evoked by the combination's constituents, and (ii) accessibility of the concepts, i.e., the activation and mobilization of those concepts and their properties for the purposes of building a new concept from the two constituents.

The principal goal of the present experiment involving the selection rather than production of interpretations was thus to see how the availability in memory of the concepts evoked by constituents N1 and N2 of conceptual combinations affects their interpretation. This issue was addressed by making relation- and property-based interpretations equally accessible to the participants. The interpretations proposed were the ones found most frequently in the results of the interpretation-production experiment conducted earlier (Jhean-Larose et al. 2009).

The main hypotheses of this experiment pertain to how the order of occurrence of the constituents (N2N1 (Head-Mod) vs. N1N2 (ModHead)), the type of conceptual combination (relation or property), and the participant's age affect the syntax (N2N1 vs. N1N2) and the type (relation vs. property) of the interpretation chosen.

If interpretations are based on the semantic nature of the constituents, and if we assume that in French (unlike in English) the principal interpretation syntax is N2N1, then regardless of the constituents' order of occurrence, N2N1 interpretations will be predominant and will increase in number with age until adulthood.

If, as assumed in the CARIN model (Gagné-Shoben 1997; Gagné $2000 ; 2001 ; 2002)$, interpreting a combination consists of construing a relation between the constituents, we can hypothesize that, younger children might give more general responses (e.g., cotton snow is 'snow that is somehow like cotton' and that older children and adults might more clearly identify (e.g., cotton snow is snow that is 'like cotton in that it is soft'). This prediction follows from Gagné (2002) which found that the salience of particular properties influences ease of interpretation for two word compounds.

If, as assumed in the dual-process model (Wisniewski 1997; Wisniewski-Clancy 2004; Wisniewski-Love 1998; Wisniewski-Middleton 2002), the first step in the interpretation of a combination consists of finding 
a scenario that relates the constituents to each other, then for relation combinations such a relation will be found. For property combinations, difficulty or failure in finding a relation (steps 2 and 3 of the model) will cause a property of one constituent to be transferred to the other.

If the accessibility of the concepts evoked by constituents $\mathrm{N} 1$ and $\mathrm{N} 2$ plays a minor role in the production task, or if it plays a similar role among children and adults, then the response pattern in the present selection task will be the same as that found earlier on the production task (Jhean-Larose et al. 2009). If, on the other hand, differences of accessibility exist between groups of children, and between children and adults, then the response patterns will not be the same: when property combinations rather than relation combinations are being interpreted, the proportion of $\mathrm{N} 2 \mathrm{~N} 1$ responses will be smaller and the proportion of property-based responses will be larger. This difference will be greater among younger children than among older ones and adults.

\section{Method}

\subsection{Participants}

One hundred and fifty-five pupils from a Parisian elementary school ${ }^{4}$ participated in the experiment. Five children failed to respond to all combinations, so their answers were not included in the analyses. The remaining 150 participants were divided into three age groups: 50 first graders (mean age $=6.8$ years), 50 third graders (mean age $=8.7$ years) and 50 fifth graders (mean age $=10.8$ years). Two adult groups of 28 sophomore students majoring in psychology at Paris XIII University also participated for course credit. All participants were native speakers of French.

\subsection{Materials and experimental setup}

Two sets of combinations were generated from Gagné ${ }^{5}$ and Shoben's (1997) lists of 'modifiers' and 'head nouns' (see Appendix A). The first set

${ }^{4}$ We would like to thank the principal of La Fontaine School and her colleagues for their collaboration.

${ }^{5}$ We are grateful to Christina Gagné for providing us with the modifier and headnoun corpora she compiled in collaboration with Edward Shoben. 
was composed of 12 combinations likely to trigger an interpretation based on a relation between the two constituents (relation combinations) and the second set was composed of 12 other combinations likely to trigger an interpretation based on the transfer of a property from one constituent to the other (property combinations). Relation combinations and property combinations were items that were 'relation' or 'property' from a child perspective (Jhean-Larose et al. 2007; 2009). This a priori assignment to the relation or property category was confirmed by the results of a preliminary test run on 22 adults (students at the Teachers' College of Paris) and 24 ten- and eleven-year-old pupils; the most frequent responses for the relation and property combinations were relation-based and property-based interpretations, respectively. The materials thus consisted of a total of 24 combinations. There was an equal number of combinations for each of six predefined relations and for each of six transfers of different properties. The presentation of the items was randomized.

The combinations in both categories exhibited low semantic similarity, as estimated by Latent Semantic Analysis (Landauer-Dumais 1997) on the TextEnfant corpus (Denhière et al. 2006; 2007). The semantic links (as measured by the mean of the cosine between the terms in each pair) for the relation and property combinations were equal to .16 and .06 , respectively.

The response choices were taken from the results of the interpretation-production task on the same combinations (see Appendix B). For a given combination, the semantic-relation sentence and the propertytransfer sentence most often produced by the children in the N2 N1 (Head-Mod) order and in the N1N2 (Mod-Head) order were used.

The participants were randomly assigned to one of two orders: O1 (N2N1, Head-Mod) or O2 (N1N2, Mod-Head), where the 'modifier' and 'head noun' categories were the ones assigned to the nouns by Gagné and Shoben (1997). Twenty-five children per age group and 28 adults were assigned to each order.

\subsection{Procedure}

Each age group was tested separately and collectively. Both children and adults were given the 24 combinations printed on two pages (front and back) where they had to check the answer selected. The two types of combinations (relation and property) were alternated. For each combination, 
the participants were instructed to pick the sentence they thought 'best expressed the connection between the two words'

The experiment began with a four-trial practice phase consisting of two relation combinations and two property combinations. The relation combinations were moto garage ('motorcycle garage') and pull laine ('pullover wool'), and the property combinations were chaussette fromage ('sock cheese') and danseuse papillon ('dancer butterfly'). No time limit was set for responding. The task lasted 20 to 30 minutes, depending on the age group.

\subsection{Response coding and data analysis}

The data were analyzed using the PAC (Program for the Analysis of Comparisons: Lecoutre-Poitevineau 2005). Separate ANOVA were conducted for each combination of the two orders of occurrence and of the two types of combination (relations and properties). Each of these analyses involved a 4 groups (Age with four modalities) analysis of variance with 6 repeated measures ( 6 stimulus items). Two dichotomous variables (1 vs. 0) were processed: interpretation syntax (with N2N1 interpretations coded as 1) and interpretation type (with relation-based interpretations coded as 1). These two variables give for each group the proportions of N2N1 (Head-Mod) responses and relation-based responses, respectively (averaged across the 6 stimulus items).

Usual ANOVA F-tests were completed by fiducial Bayesian procedures (Rouanet 1996; Lecoutre 2006; 2008). These procedures give interval estimates for each population proportion $p$ and allow to estimate the magnitude of the differences between two proportions. In most situations, we are able to assess the practical significance of an observed difference between two proportions, that is, to answer questions such as "is this observed difference $D$ notable (large enough) or negligible (small enough)?" Unfortunately, the statistical significance of the ANOVA $p$ value only address questions about the existence of the population difference $\delta$ and says nothing about its size. On the one hand, even a "highly significant" test ( $p$ "very small") only establishes that the null hypotheses $d=0$ is rejected. On the other hand, a "non-significant" test is only a failure to reject this hypothesis and is generally considered as non-conclusive. Consequently, significance tests must be completed with statistical inference procedures that answer questions about the practical 
significance of the population difference: "is $\delta$ large or small?" Fiducial Bayesian procedures directly answer such questions. For each difference of interest, we report the observed difference-for instance, $D=0.220$ (large) - the associated $F$-test and $p$ value $-F(1,198)=30.3, p<0.0001$ (significant) - and an inferential statement about the population difference $d$-here $d>0.160$ - associated with a probability (or credibility) $90 \%$. In this case, we can conclude that the population difference is large (larger than 0.160 with probability $90 \%$ ). Fiducial Bayesian procedures are closely related to the $F$ - and $t$-tests (Lecoutre et al. 2010): $1-p / 2$ is the fiducial Bayesian probability of a same-sign difference in the population (here $d$ is positive with probability $>99.99 \%$ ). This interpretation of the one-tailed $p$ value $(p / 2)$ can be viewed as the counterpart of the Jones and Tukey (2000) view of null hypothesis significance tests as a three-alternative decision: the sign is positive, is negative, or is not yet determined. Moreover, an important feature is that the $100(1-d) \%$ fiducial-Bayesian interval estimate of $d$ (i.e., a $100(1-a) \%$ equal-tails probability interval centered on $D$ ) coincide with the usual confidence interval associated with the ANOVA $F$ ratio.

\section{Results}

\subsection{Results of the selection experiment}

\subsubsection{Interpretation syntax: Analysis of Head-Mod responses}

Table 1 presents the proportions of Head-Mod responses.

\subsubsection{Order of occurrence Head-Mod}

On relation combinations, there were more Head-Mod responses $[0.573$, $0.548<p<0.598]$ than responses with the opposite syntax. The proportion of Head-Mod responses was significantly greater for adults: 0.679 vs. $0.533, F(1,99)=18.6, p<0.0001, D=0.145, d>0.102$. Among children, the proportions increased with age; there was a significant linear trend: $F(1,72)=10.1, p<0.002$, the observed slope of the regression line being $D=0.030(d>0.018)$.

On property combinations, there were less Head-Mod responses than Mod-Head responses for children [0.429, $0.401<p<0.457$ ], while the proportion of these responses was especially high for adults [0.839, 
Table 1

Observed proportion of Head-Mod responses for the four groups of subjects, for each type of combination and each order of occurrence (90\% interval estimates in brackets)

\begin{tabular}{lcccccc}
\hline & \multicolumn{2}{c}{ Order O1: Head-Mod } & & \multicolumn{2}{c}{ Order O2: Mod-Head } \\
\cline { 6 - 6 } \cline { 6 - 6 } & $\begin{array}{c}\text { Relation } \\
\text { combinations }\end{array}$ & $\begin{array}{c}\text { Property } \\
\text { combinations }\end{array}$ & & $\begin{array}{c}\text { Relation } \\
\text { combinations }\end{array}$ & $\begin{array}{c}\text { Property } \\
\text { combinations }\end{array}$ \\
\hline First graders & 0.48 & 0.38 & & 0.51 & 0.45 \\
& {$[0.44,0.53]$} & {$[0.31,0.45]$} & & {$[0.45,0.56]$} & {$[0.38,0.51]$} \\
\hline Third graders & 0.51 & 0.45 & & 0.54 & 0.45 \\
& {$[0.48,0.55]$} & {$[0.40,0.50]$} & {$[0.48,0.60]$} & {$[0.41,0.49]$} \\
\hline Fifth graders & 0.60 & 0.46 & & 0.52 & 0.53 \\
& {$[0.55,0.66]$} & {$[0.40,0.52]$} & {$[0.46,0.58]$} & {$[0.46,0.61]$} \\
\hline Adults & 0.68 & 0.84 & & 0.35 & 0.62 \\
& {$[0.62,0.74]$} & {$[0.80,0.88]$} & {$[0.28,0.43]$} & {$[0.53,0.70]$} \\
\hline Pooled & 0.57 & 0.54 & 0.48 & 0.51 \\
& {$[0.55,0.60]$} & {$[0.52,0.56]$} & {$[0.45,0.51]$} & {$[0.48,0.55]$} \\
\hline
\end{tabular}

$0.803<p<0.876]$. So the difference between adults and children was particularly notable: $F(1,99)=181.1, p<0.0001, D=0.410, d>0.371$.

\subsubsection{Order of occurrence Mod-Head}

On relation combinations, the proportion of Head-Mod responses for children was about $0.50[0.522,0.489<p<0.556]$, while it was smaller than 0.50 for adults $[0.354,0.281<p<0.427]$. The difference between children and adults was significant: $F(1,99)=15.9, p=0.0001, D=0.168$, $d>0.114$.

On property combinations, the proportion of Head-Mod responses for children was again about 0.50 [0.477, $0.448<p<0.506]$, while it was larger than 0.50 for adults $[0.619,0.549<p<0.689]$. The difference between adults and children was significant: $F(1,99)=14.1, p=0.0003$, $D=0.142, d>0.093$. Among children, the proportions increased with age; there was a significant linear trend: $F(1,72)=4.20, p=0.04$, the observed slope of the regression line being $D=0.022(d>0.008)$. 


\subsubsection{Conclusion}

The results obtained in the two orders of occurrence were relatively comparable among children, if we except the increase with age observed for Relation combinations only observed for the order Head-Mod. On the contrary, for adults the proportion of Head-Mod responses was found to be markedly smaller for the Mod-Head order, for each type of combinations.

\subsubsection{Interpretation type: Analysis of relation-based responses}

Table 2 gives the proportions of responses based on a relation between the two constituents.

\section{Table 2}

Observed proportion of relation-based responses for the four groups of subjects, for each type of combination and each order of occurrence (90\% interval estimates in brackets)

\begin{tabular}{lccccc}
\hline & \multicolumn{2}{c}{ Order O1: Head-Mod } & & \multicolumn{2}{c}{ Order O2: Mod-Head } \\
\cline { 6 - 6 } \cline { 6 - 6 } & $\begin{array}{c}\text { Relation } \\
\text { combinations }\end{array}$ & $\begin{array}{c}\text { Property } \\
\text { combinations }\end{array}$ & & $\begin{array}{c}\text { Relation } \\
\text { combinations }\end{array}$ & $\begin{array}{c}\text { Property } \\
\text { combinations }\end{array}$ \\
\hline First graders & 0.71 & 0.42 & & 0.67 & 0.46 \\
& {$[0.65,0.77]$} & {$[0.37,0.47]$} & & {$[0.61,0.73]$} & {$[0.39,0.42]$} \\
\hline Third graders & 0.69 & 0.41 & & 0.68 & 0.48 \\
& {$[0.63,0.75]$} & {$[0.36,0.47]$} & {$[0.61,0.75]$} & {$[0.41,0.55]$} \\
\hline Fifth graders & 0.73 & 0.51 & 0.70 & 0.43 \\
& {$[0.67,0.79]$} & {$[0.44,0.58]$} & {$[0.64,0.75]$} & {$[0.34,0.51]$} \\
\hline Adults & 0.58 & 0.16 & 0.56 & 0.18 \\
& {$[0.50,0.66]$} & {$[0.12,0.20]$} & {$[0.50,0.62]$} & {$[0.13,0.24]$} \\
\hline Pooled & 0.67 & 0.37 & 0.65 & 0.38 \\
& {$[0.64,0.71]$} & {$[0.34,0.40]$} & {$[0.62,0.68]$} & {$[0.35,0.42]$} \\
\hline
\end{tabular}

\subsubsection{Order of occurrence Head-Mod}

On relation combinations, there were more Head-Mod responses $[0.674$, $0.647<p<0.701]$ than responses with the opposite syntax. Among children, the proportion of Head-Mod responses was clearly larger than half for the three age groups, the smaller proportion being observed for 
third graders $[0.690,0.640<p<0.740]$. It was significantly greater than for adults: 0.710 vs. $0.577, F(1,99)=12.9, p=0.0005, D=0.133$, $d>0.085$.

On property combinations, there were less Head-Mod responses than Mod-Head responses [0.370, $0.348<p<0.392]$. The proportion of HeadMod responses was especially weak for adults [0.158, $0.124<p<0.191]$. It was significantly smaller than for children: 0.449 vs. $0.158, F(1,99)=$ $96.2, p<0.0001, D=0.291, d>0.253$.

\subsubsection{Order of occurrence Mod-Head}

On relation combinations, there were more of relation-based responses $[0.647,0.622<p<0.673]$ than of property-based responses. Among children, the proportion of relation-based responses was clearly larger than half for the three age groups, the smaller proportion being observed for first graders [0.667, $0.615<p<0.718]$. It was significantly greater than for adults: 0.680 vs. $0.560, F(1,99)=12.1, p=0.0007, D=0.120$, $d>0.076$.

On property combinations, there were fewer relation-based responses than property-based responses [0.383, $0.354<p<0.411]$. The proportion of relation-based responses was especially weak for adults $[0.185$, $0.139<p<0.230]$. It was significantly smaller than for children: 0.457 vs. $0.185, F(1,99)=50.1, p<0.0001, D=0.272, d>0.223$.

\subsubsection{Conclusion}

The results obtained in the two orders of occurrence were comparable for all groups of subjects.

\subsection{Comparison of selection and production experiments}

Interval estimates for proportions were used to compare the two experiments. For each proportion of interest, we report the observed proportion $P$ and the $90 \%$ interval estimate for the parent proportion $p$. These proportions are indexed by $S$ and $P$, respectively for the selection and production experiments (for instance, $P_{S}=0.526,0.512<p_{S}<0.541$ ). 


\subsubsection{Interpretation syntax: Analysis of Head-Mod responses}

Head-Mod interpretations strongly outnumbered interpretations with the opposite syntax on the production task $\left[P_{P}=0.690,0.675<p_{P}<0.705\right]$, and did so slightly on the selection task $\left[P_{S}=0.526,0.512<p_{S}<0.541\right]$. This difference was particularly large in the Head-Mod order $\left[P_{P}=0.788\right.$, $0.772<p_{P}<0.803$ vs. $\left.P_{S}=0.557,0.539<p_{S}<0.574\right]$, and relatively smaller in the Mod-Head order $\left[P_{P}=0.588,0.563<p_{P}<0.613\right.$ vs. $\left.P_{S}=0.557,0.496<p_{S}<0.519\right]$.

This difference was large both for relation combinations $\left[P_{P}=0.707\right.$, $0.690<p_{P}<0.724$ vs. $\left.P_{S}=0.525,0.505<p_{S}<0.545\right]$, and for property combinations $\left[P_{P}=0.673,0.655<p_{P}<0.690\right.$ vs. $P_{S}=0.528,0.510<$ $\left.p_{S}<0.546\right]$.

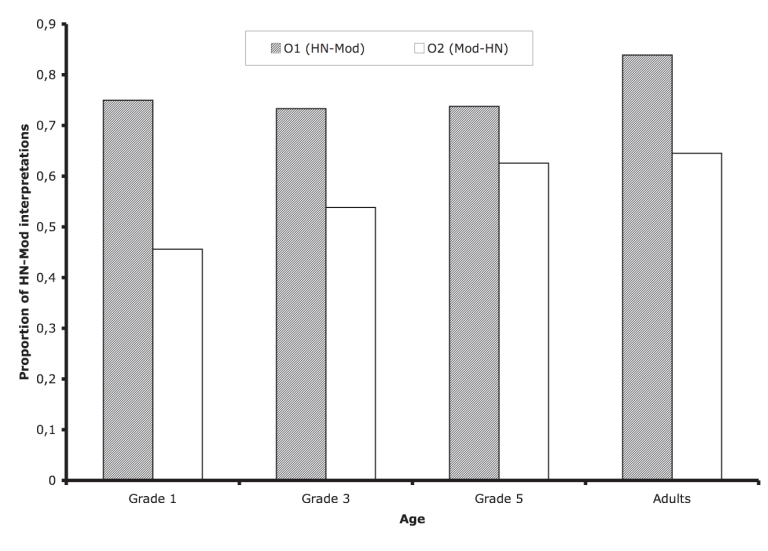

Fig. 1

Proportion of Head-Mod interpretations, by age and by order of constituent occurrence

On relation combinations, the difference between production and selection was also large, both for the children $\left[P_{P}=0.681,0.661<p_{P}<0.701\right.$ vs. $\left.P_{S}=0.528,0.507<p_{S}<0.549\right]$ and for the adults $\left[P_{P}=0.737\right.$, $0.708<p_{P}<0.765$ vs. $\left.P_{S}=0.516,0.470<p_{S}<0.563\right]$.

On property combinations, on the other hand, the difference between production and selection was large only for the children $\left[P_{P}=0.600\right.$, $0.578<p_{P}<0.622$ vs. $\left.P_{S}=0.453,0.433<p_{S}<0.473\right]$. 


\subsubsection{Interpretation type: Analysis of relation-based responses}

Relation-based responses, which outnumbered the others in both tasks, were more numerous in production $\left[P_{P}=0.656,0.646<p_{P}<0.667\right]$ than in selection $\left[P_{S}=0.518,0.506<p_{S}<0.531\right]$. The difference was large in the two orders: Head-Mod $\left[P_{P}=0.658,0.642<p_{P}<0.673\right.$ vs. $\left.P_{S}=0.522,0.506<p_{S}<0.538\right]$ and Mod-Head $\left[P_{P}=0.655,0.642<\right.$ $p_{P}<0.669$ vs. $\left.P_{S}=0.515,0.495<p_{S}<0.535\right]$.

The production vs. selection difference was large for relation combinations $\left[P_{P}=0.883,0.876<p_{P}<0.890\right.$ vs. $P_{S}=0.661,0.642<$ $\left.p_{S}<0.679\right]$, and was of limited importance for property combinations $\left[P_{P}=0.430,0.412<p_{P}<0.448\right.$ vs. $\left.P_{S}=0.376,0.358<p_{S}<0.394\right]$.

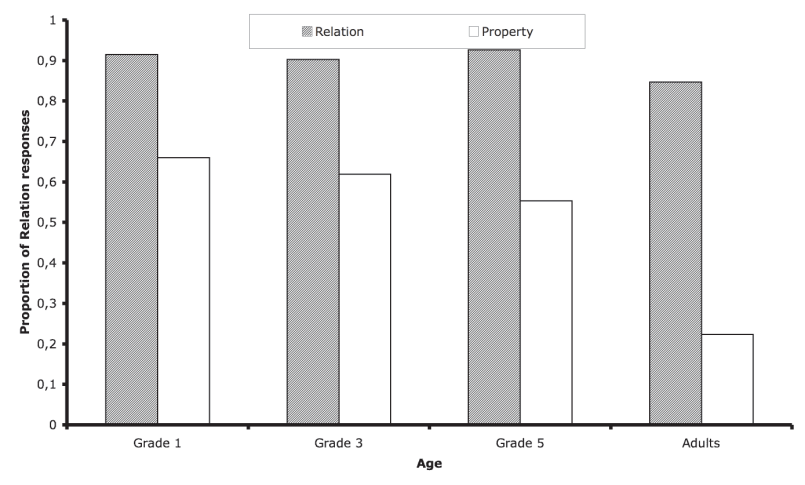

Fig. 2

Proportion of Relation responses, by age and by type of combinations

On relation combinations, the difference between production and selection was large, both for the children $\left[P_{P}=0.915,0.908<p_{P}<0.922\right.$ vs. $\left.P_{S}=0.695,0.675<p_{S}<0.715\right]$ and for the adults $\left[P_{P}=0.847\right.$, $0.833<p_{P}<0.860$ vs. $\left.P_{S}=0.568,0.527<p_{S}<0.610\right]$.

On property combinations, the difference between production and selection was large only for the children $\left[P_{P}=0.612,0.588<p_{P}<0.860\right.$ vs. $\left.P_{S}=0.453,0.430<p_{S}<0.475\right]$. In short, the following main points stand out from our comparison of the production and selection experiments. 


\section{Discussion}

This selection experiment was aimed at determining how the availability in memory of the two concepts evoked by the first (N1) and second (N2) constituents of noun-noun combinations affects the interpretation of such combinations. Earlier production-task results (for both the syntax and type of the interpretations made; Jhean-Larose et al. 2009) were replicated as a whole, but to a lesser extent. Our hypothesis - that relation-based interpretations would prevail for relation combinations and property-based interpretations would prevail for property combinations - was also validated, but with differences between children and adults: on relation combinations, relation-based interpretations were more numerous for children than for adults, whereas on property combinations, relation-based interpretations were outnumbered by property-based ones to a lesser extent among the children than among the adults.

Comparison of the results obtained on the selection and production experiments showed that providing equal accessibility to the four most-frequent interpretations produced by children, triggered different effects on both child and adult performance. The effects of the order-ofoccurrence and type-of-combination factors on the two dependent variables, interpretation syntax and type, differed across tasks, and the childadult differences varied with the type of combination. These interactions confirm the existence of differing effects of the availability of constituent concepts on the production and selection of interpretations. In a recent work with adults, Spalding et al. (2010) found that the head noun did have an influence when people were asked to verify a relation (e.g., mountain bird: a bird in the mountains) but not when people did a sense/ nonsense judgment (e.g., judging whether mountain bird has a plausible interpretation).

\section{Conclusion}

Our first objective was to answer the question raised here about the interpretation order of novel noun-noun conceptual combinations: Do French speakers process these combinations in the N1N2 (Mod-Head) order, as English speakers do, or in the N2N1 (Head-Mod) order as suggested in some linguistic studies (Noailly 1990)? 
Our second objective concerned the influence of the type of combination on the interpretations made. We defined two combination categories that differed as to the nature of the most probable connection between the two constituents, i.e., a relation (Gagné-Shoben 1997) or a property (Wisniewski 1997). The idea was to determine whether - as predicted by Gagné and Shoben (1997) - all interpretations are based on a construed relation between the two constituents, or whether some interpretations are based on the transfer of a property from one constituent to the other whenever a relation-based interpretation is difficult or impossible (Wisniewski 1997).

To answer these questions, we compared the performance of adults and three groups of children ages 6,8 , and 10, insofar as earlier research has shown that children have trouble transferring properties from one constituent to the other (Parault et al. 2005). We also wanted to find out how the accessibility and availability in memory of the constituent concepts affect the interpretation of conceptual combinations. To test for this, we compared the results of two experiments that differed in the extent to which memory-accessibility processes were involved: selection versus production, with selection bearing on the four most-frequently-produced responses to combinations obtained by crossing the order-of-occurrence (N2N1 or N1N2) and type-of-combination (relation or property) factors.

The results obtained provided a clear-cut answer to the first question. French speakers, both children and adults, act differently from English speakers: they most often interpret novel noun-noun combinations in the N2N1 (Head-Mod) order given the syntactic characteristics of the French language, where the epithet noun (the modifier) comes after the head noun.

Based on the findings of the selection experiment and on the findings of the comparison of selection and production experiments, we can legitimately conclude, then, that both children and adults primarily produce and select a relation-based interpretation for relation combinations. However, the proportion of relation-based responses was considerably lower for selecting than for producing interpretations, which shows that concept availability in memory has an impact. The availability effect was also noted for property combinations, since relation-based responses predominated in the production task but not in the selection task. While the adults gave mainly relation-based responses for relation combinations and property-based responses for property combinations, in line with our hypothesis, the children did so only in the selection experi- 
ment. The observed age-related change in the proportion of relation-based responses varied across combination types, with relative stability for relation combinations and a variable, task-dependent decrease for property combinations.

Our data support the CARIN theory and are incompatible with the dual-process theory. First, the modifier and head noun constituents in the relation and property conditions were equated for semantic similarity and, importantly, both have low semantic similarity. Yet, one of the main claims of the dual-process theory is that property interpretations are only attempted when the constituents are highly semantically related. The finding that property interpretations are produced for these materials contradicts one of the major predictions of this theory. That is, degree of similarity between the two constituent nouns does not appear to be predictive of whether property-mapping is used and this finding alone undermines the dual-process theory. Some of the other literature also fails to support Wisniewski's prediction that there is a strong relationship between similarity and the likelihood of using property-mapping. Increased similarity leads to more hybrid interpretations (e.g., statements that a $\mathrm{N} 1 \mathrm{~N} 2$ is something that is both a $\mathrm{N} 1$ and a N2: a kidnapper killer is both a killer and a kidnapper), rather than to property mappings. This evidence is inconsistent with the idea that increased similarity tends to increase property usage. For example, Downing (1977) notes that highly similar pairs (e.g., pie cake) were judged to be uninterpretable. Likewise, Gagné (2000) found that the most common response to highly similar combinations was a non-combination strategy: $48 \%$ of these items were interpreted as being "both members" of a category. For example, whiskey beer was interpreted using a property or a relation. This suggests that increased similarity does not always lead to increased use of property mapping. Bock and Clifton (2000) also failed to find an effect of similarity on interpretation: there was no correlation between the proportion of property mapping interpretations and similarity.

Second, the dual-process theory has no explanation for why the proportion of interpretations would vary with the task (selection vs. production) because the semantic similarity of the constituents remain constant in both tasks. Third, the explanation put forth for the reason why the proportion of property interpretations does not coincide with the main claims of the dual-process theory. The claim is that children are able to align the properties of the two constituents but have trouble selecting which properties to map. However, if children do indeed align 
the properties (step 1), then they would not be producing relation-based interpretations. The theory claims that people attempt either propertymapping or relation-linking. Failed attempts at property-mapping would result in unsuccessful interpretations, not in relation-based interpretations. If children were having trouble with steps 2 and 3 (transfer and incorporation of properties), then these cases would still be examples of property-interpretations and the proportion of property to relation interpretations would not change with age. Finally, a primary implication of the dual-process theory is that both routes are equally easy. If so, then why should property-mapping be more difficult? Also, if anything, it would seem that relation-linking which requires more abstract generalizations than property-mapping should be more difficult for children.

The change in proportion of property-interpretations for propertyitems is more compatible with the CARIN theory because this theory argues that people initially create a relation-based explanation (e.g., neige coton: 'snow that is like cotton') and then, in some cases, use this structure to determine properties of the new structure (e.g., 'snow that is like cotton is very white, soft, puffy, etc.'). Thus, it could be the case that older children are better able to settle on an appropriate relation and then elaborate the resulting combined concept by mentioning particular properties. Gagné (2000) showed that property interpretations take longer to come up with than relation interpretations and this suggests that they might be occurring after the construction of a relation-based interpretation.

\section{References}

Bock, John - Charles Clifton 2000. The role of salience in conceptual combination. In: Memory and Cognition 28:1378-86.

Bowdle, Brian - Dedre Gentner 2005. The career of metaphor. In: Psychological Review $112: 193-216$.

Caramelli, Nicoletta - Anna Borghi 2004. The role of Head Nouns in conceptual combination. In: Jean Drévillon - Jean Vivier - Agnès Salinas (eds): Psycholinguistics: A multidisciplinary science. Proceedings of the VIth ISAPL International Congress, Caen, June 28-July 1 2000, 87-92. Europia, Paris.

Costello, Fintan-Mark Keane 2000. Efficient creativity: Constraint-guided conceptual combination. In: Cognitive Science $24: 299-349$. 


\section{SANDRA JHEAN-LAROSE-BRUNO LECOUTRE-GUY DENHIÈRE}

Costello, Fintan-Mark Keane 2001. Testing two theories of conceptual combination: Alignment vs diagnosticity in the comprehension and production of combined concepts. In: Journal of Experimental Psychology: Learning, Memory \& Cognition $27: 255-71$.

Denhière, Guy - Cedrick Bellissens - Sandra Jhean-Larose - Benoît Lemaire 2007. A semantic space for modeling a child's semantic memory. In: Thomas K. LandauerDanielle S. McNamara - Simon Dennis - Walter Kintsch (eds): Handbook of latent semantic analysis, 143-65. Lawrence Erlbaum, Mahwah NJ.

Denhière, Guy-Benoît Lemaire-Cedrick Bellissens-Sandra Jhean-Larose 2006. A computational model for simulating text comprehension. In: Behavior Research Methods 38:628-37.

Downing, Pamela 1977. On the creation and use of English compound nouns. In: Language $53: 810-42$.

Estes, Zachary - Sam Glucksberg 1999. Relevance and feature accessibility in combined concepts. In: Martin Hahn-Scott C. Stoness (eds): Proceedings of the 21st Annual Conference of the Cognitive Science Society, 149-54. Erlbaum, Mahwah NJ.

Estes, Zachary-Sam Glucksberg 2000. Interactive property attribution in concept combination. In: Memory and Cognition 28:28-34.

Gagné, Christina 2000. Relation-based combinations versus property-based combinations: A test of the CARIN theory and dual-process theory of conceptual combination. In: Journal of Memory and Language 42:365-89.

Gagné, Christina 2001. Relation and lexical priming during the interpretation of nounnoun combinations. In: Journal of Experimental Psychology: Learning, Memory \& Cognition $27: 236-54$

Gagné, Christina 2002. Lexical and relational influences on the processing of novel compounds. In: Brain and Language $81: 723-35$.

Gagné, Christina - Edward Shoben 1997. Influence of thematic relations on the comprehension of modifier-noun combinations. In: Journal of Experimental Psychology: Learning, Memory \& Cognition 23:71-87.

Gentner, Dedre 1983. Structure-mapping: A theoretical framework for analogy. In: Cognitive Science $7: 155-70$.

Gentner, Dedre 1989. The mechanisms of analogical learning. In: Stella VosniadouAndrew Ortony (eds): Similarity and analogical reasoning, 199-241. Cambridge University Press, Cambridge.

Gineste, Marie-Dominique-Véronique Scart-Lhomme 1999. Comment comprenonsnous les métaphores? In: L'Année Psychologique 99: 447-92.

Glucksberg, Sam-Matthew McGlone-Deanna Manfredi 1997. Property attribution in metaphor comprehension. In: Journal of Memory and Language $36: 50-67$.

Hampton, James 1987. Inheritance of attributes in natural concept conjunctions. In: Memory and Cognition 15:55-71.

Jhean-Larose, Sandra-Guy Denhière 2006. Etude des processus cognitifs d'interprétation de combinaisons conceptuelles nouvelles. In: L'Année Psychologique 2: $265-304$.

Acta Linguistica Hungarica 58, 2011 
Jhean-Larose, Sandra-Guy Denhière 2007. Word order and type of relation effects in conceptual combination during the early school years. In: Stella VosnadiouDaniel Kayser-Athanassios Protopapas (eds): Proceedings of the EuroCogSci 07, The European Cognitive Science Conference 2007, Delphes, 919. Psychology Press, Hove.

Jhean-Larose, Sandra-Guy Denhière-Bruno Lecoutre 2009. Étude développementale de l'interprétation de combinaisons conceptuelles nominales « Relation » et « Propriété ». In: Psychologie Française $54: 121-35$.

Jhean-Larose, Sandra-Guy Denhière-Fabiola Martinez 2007. A developmental approach of word order and type of relation effects in conceptual combination. Paper presented at the British Psychological Society, Developmental Section, University of Plymouth, 29-31 August 2007.

Jones, Lyle V.- John W. Tukey 2000. A sensible formulation of the significance test. In: Psychological Methods 5:411-4.

Landauer, Tom-Suzanne Dumais 1997. A solution to Plato's problem: The Latent Semantic Analysis Theory of the acquisition, induction and representation of knowledge. In: Psychological Review 104 : 211-40.

Le Ny, Jean-François 2005. Comment l'esprit produit du sens. Odile Jacob, Paris.

Lecoutre, Bruno 2006. Training students and researchers in Bayesian methods for experimental data analysis. In: Journal of Data Science 4:207-32.

Lecoutre, Bruno 2008. Bayesian methods for experimental data analysis. In: Calyampudi Radhakrishna Rao-J. Philip Miller-Dabeern C. Rao (eds): Handbook of statistics: Epidemiology and medical statistics (Vol. 27), 775-812. Elsevier, Amsterdam.

Lecoutre, Bruno - Marie-Paule Lecoutre - Jacques Poitevineau 2010. Killeen's probability of replication and predictive probabilities: How to compute, use and interpret them. In: Psychological Methods $15: 158-71$.

Lecoutre, Bruno-Jacques Poitevineau 2005. Le logiciel «LePAC» dédié aux procédures bayésiennes. In: La Revue Modulad 33. (http://tinyurl.com/chfuhkh)

Levi, Judith 1978. The syntax and semantics of complex nominals. Academic Press, New York.

Markman, Arthur B.-Dedre Gentner 1993. Structural alignment during similarity comparisons. In: Cognitive Psychology 25:431-67.

Markman, Arthur B.-Edward J. Wisniewski 1997. Similar and different: The differentiation of basic level categories. In: Journal of Experimental Psychology: Learning, Memory, and Cognition 23:54-70.

Medin, Douglas - Edward Shoben 1988. Context and structure in conceptual combination. In: Cognitive Psychology $20: 158-90$.

Murphy, Gregory L. 1988. Comprehending complex concepts. In: Cognitive Science $12: 529-62$.

Murphy, Gregory L. 1990. Noun phrase interpretation and conceptual combination. In: Journal of Memory and Language $29: 259-88$.

Murphy, Gregory L. 2002. The big book of concepts. MIT Press, Cambridge MA.

Noailly, Michèle 1990. Le substantif épithète. Presses Universitaires de France, Paris. 


\section{SANDRA JHEAN-LAROSE-BRUNO LECOUTRE-GUY DENHIÈRE}

Parault, Susan-Paul Schwanenflugel-Heather Rogers Haverback 2005. The development of interpretations for novel noun-noun conceptual combinations during the early school years. In: Journal of Experimental Psychology $91: 67-87$.

Rouanet, Henri 1996. Bayesian methods for assessing importance of effects. In: Psychological Bulletin 119:149-58.

Spalding, Thomas - Christina Gagné - Allison Mullaly - Hongbo Ji 2010. Relation-based interpretations of noun-noun phrases: A new theoretical approach. In: Linguistische Berichte, Sonderheft $17: 283-315$.

Storms, Gert-Edward Wisniewski 2005. Does the order of head noun and modifier explain response times in conceptual combination? In: Memory and Cognition $33: 852-61$.

Tiberghien, Guy 1997. La mémoire oubliée. Mardaga, Liège.

Wisniewski, Edward 1996. Construal and similarity in conceptual combination. In: Journal of Memory and Language $35: 434-53$

Wisniewski, Edward 1997. When concepts combine. In: Psychonomic Bulletin \& Review $4: 167-83$.

Wisniewski, Edward-Emily J. Clancy 2004. You don't need a weatherman to know which way the wind blows: The role of discourse context in conceptual combination. Unpublished manuscript.

Wisniewski, Edward - Bradley C. Love 1998. Properties versus relations in conceptual combination. In: Journal of Memory and Language 38:177-202.

Wisniewski, Edward-Erica Middleton 2002. Of bucket bowls and coffee cup bowls: Spatial alignment in conceptual combination. In: Journal of Memory and Language $46: 1-23$.

Wolff, Phillip-Dedre Gentner 2000. Evidence for role-neutral initial processing of metaphors. In: Journal of Experimental Psychology: Learning, Memory, and Cognition 26:1-13. 


\section{Appendix A}

Relation-based and property-based conceptual combinations taken from Gagné and Shoben's (1997) modifier and head-noun corpora:

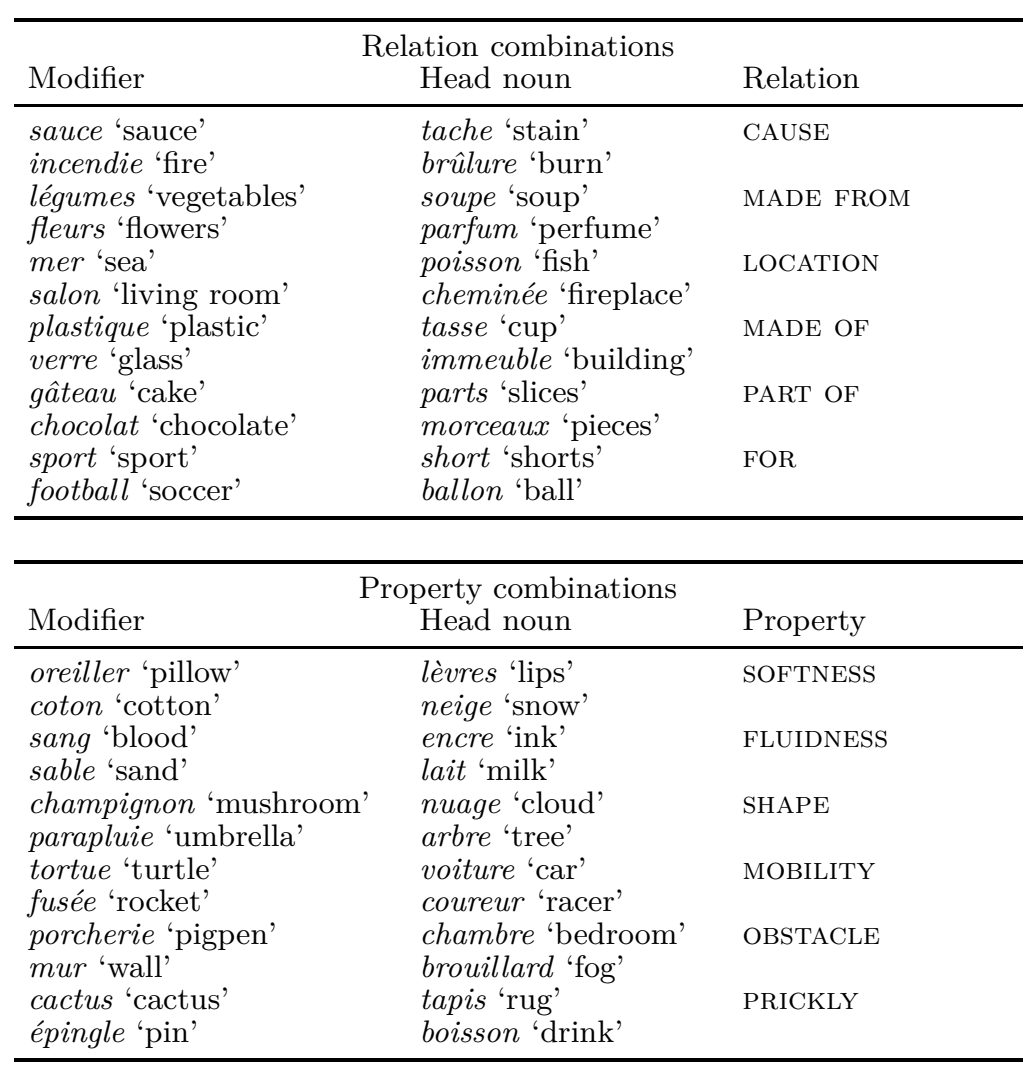




\section{SANDRA JHEAN-LAROSE-BRUNO LECOUTRE-GUY DENHIÈRE}

\section{Appendix B}

Example of the selection-task materials. O1 stands for the N2N1 'Head-Mod' order of occurrence, $\mathrm{O} 2$ for the opposite order. P stands for property transfer, $\mathrm{R}$ for construed relation between the two constituents. Again, the four sentences used corresponded to the ones produced most often on the production task (see Jhean-Larose et al. 2009).

Property combination: voiture tortue 'car turtle' (Order N2N1 Head-Mod)

01P1: La voiture roule lentement comme une tortue.

'The car moves slowly like a turtle'

02P2: Une tortue marche vite comme une voiture.

'A turtle moves fast like a car'

O1R1: Cette voiture ressemble à une tortue.

'This car is like a turtle'

O2R2: Je mets ma tortue dans la voiture.

'I put my turtle in the car'

Relation combination: cheminée salon 'fireplace living room' (Order N2N1 Head-Mod)

01P1: La cheminée est grande comme un salon.

'The fireplace is as big as a living room'

02P2: Le salon est poussiéreux comme la cheminée. 'The living room is dusty like the fireplace'

01R1: La cheminée est dans le salon.

'The fireplace is in the living room'

O2R2: Dans le salon, il y a une cheminée.

'In the living room, there is a fireplace' 